\title{
Low-molecular weight fucoidan inhibits the differentiation of osteoclasts and reduces osteoporosis in ovariectomized rats
}

\author{
XIN JIN $^{1}$, LIGUO ZHU ${ }^{2}$, XIULAN LI ${ }^{1}$, JIAN JIA ${ }^{1}$, YANG ZHANG ${ }^{1}$, \\ XIAOLEI SUN ${ }^{1}$, JIANXIONG MA ${ }^{1}$, ZHAOJIE LIU ${ }^{1}$ and XINLONG MA ${ }^{1}$ \\ ${ }^{1}$ Orthopedics Department, Tianjin Hospital, Tianjin 300050; ${ }^{2}$ Orthopedics Department, \\ China Academy of Traditional Chinese Medicine, Beijing 100102, P.R. China
}

Received November 5, 2015; Accepted November 7, 2016

DOI: $10.3892 / \mathrm{mmr} .2016 .6062$

\begin{abstract}
Fucoidan is a type of sulfated polysaccharide isolated from seaweed. The present study used ovariectomized Sprague-Dawley rats, which were treated with fucoidan. The effects of fucoidan on bone metabolism, density and microarchitecture were assessed using micro-computed tomography (CT), histomorphometric analysis, biochemical markers of bone metabolism (Serum procollagen type I N propeptide and C-terminal telopeptide-1) and tests of mechanical competence of the femur. In addition, the effects of low-molecular weight fucoidan (LMWF) on in vitro cultured osteoclasts were examined, in order to determine the mechanisms underlying LMWF-induced osteoclastic inhibition. In ovariectomized rats, LMWF increased femoral bone density. Micro-CT scan also revealed that LMWF prevented microarchitectural deterioration and histomorphometric analysis determined that LMWF increased trabecular bone number and reduced the surface of bone resorption. In addition, LMWF reduced the high bone turnover rate, and improved the mechanical properties of the femur in ovariectomized rats. In vitro experiments revealed that LMWF inhibited the receptor activator of nuclear factor $\kappa \mathrm{B}$ ligand (RANKL) and macrophage colony-stimulating factor-induced differentiation of RAW264.7 cells into tartrate-resistant acid phosphatase (TRAP)-positive osteoclasts, and reduced the bone resorption surface of the osteoclasts. Reverse transcription-quantitative polymerase chain reaction demonstrated that LMWF inhibited mRNA expression of TRAP, matrix metallopeptidase-9, nuclear activator of activated T-cells 1 , and osteoclast-associated immunoglobulin-like receptor, which are components of the signaling pathway for osteoclast differentiation. LMWF had no effect on RANK mRNA expression. In conclusion, the present study confirmed that LMWF inhibited osteoclast
\end{abstract}

Correspondence to: Dr Xinlong Ma, Orthopedics Department, Tianjin Hospital, 122 Munan Road, Heping, Tianjin 300050, P.R. China

E-mail: gengxiao502@163.com

Key words: osteoporosis, osteoclast, fucoidan differentiation and bone resorption, and may be a potential treatment for osteoporosis in ovariectomized rats.

\section{Introduction}

Osteoporosis is a bone disease that is characterized by reduced bone mass and microarchitectural deterioration, which may lead to an increased risk of bone fractures. It is estimated that $20 \%$ of women over 50 years old develop osteoporosis globally (1). In addition, female bone density decreases rapidly during perimenopause and continues to gradually decrease in postmenopausal women (2). Osteoporosis decreases bone strength and increases the risk of fractures $(3,4)$. Among aged individuals, hip fractures are associated with high mortality rates (5). Normal bone remodeling is achieved by a balance between bone resorption and bone formation (6); however, in postmenopausal osteoporosis, the rate of bone resorption by osteoclasts is greater compared with bone formation by osteoblasts (7). Receptor activator of nuclear factor (NF)- $\kappa \mathrm{B}$ ligand (RANKL) and macrophage colony-stimulating factor (M-CSF) are key for the maturation of osteoclasts (8). The binding of RANKL to receptor activator of nuclear factor $\kappa B$ (RANK) protein on the membrane of osteoclast precursor cells activates the NF- $\kappa \mathrm{B}$ pathway $[\mathrm{NF}-\kappa \mathrm{B}$ is the key nuclear activator of nuclear activator of activated T-cells 1 (NFATc1) expression], which may result in fusion of mononuclear osteoclasts (9). The main role of M-CSF is to induce pre-osteoclasts to express RANK, which is a receptor of RANKL (10).

At present, therapeutic strategies used to treat osteoporosis involve interference with either the differentiation of osteoclasts or their function. However, many of these drugs exert unwanted side effects. For example, bisphosphonates and denosumab have been associated with osteonecrosis of the jaw and atypical femur fractures. In addition, bisphosphonates have been associated with atrial fibrillation and kidney damage $(11,12)$. Therefore, the development of novel therapies for the treatment of osteoporosis is required.

Fucoidan is a type of sulfated polysaccharide isolated from seaweed (13). It predominantly consists of fucose, and small amounts of alduronic acid, galactose and xylose. A previous study reported that fucoidan may exhibit antitumorigenic, antiviral, anticoagulative and antioxidative properties (14). Furthermore, it has been reported that low-molecular weight 
fucoidan (LMWF) promotes the expression of alkaline phosphatase and type I collagen (15). However, the effects of LMWF on osteoclasts and osteoporosis in ovariectomized rats remain to be elucidated. A previous study reported that apoptosis induced by fucoidan was attenuated by caspase inhibitors, indicating that fucoidan-induced apoptosis was dependent on the activation of caspases (16). Fucoidan contains $\geq 25 \%$ organic sulfate and $60 \%$ polysaccharide.

A previous study compared the biological activity of LMWF and regular fucoidan, and the results indicated that LMWF had a higher activity compared with fucoidan (17). To the best of our knowledge, the effects of LMWF on osteoporosis have not been previously assessed.

The present study aimed to determine the effects and mechanisms of action of LMWF on osteoporosis, using an ovariectomized female rat model. Furthermore, the effects of LMWF on RANKL and M-CSF-induced RAW264.7 cell differentiation into osteoclasts were investigated in vitro.

\section{Materials and methods}

Preparation of LMWF. Fucoidan (Sigma-Aldrich; Merck Millipore, Darmstadt, Germany) was digested consecutively with glucoamylase, pectinase and cellulose complex (Sigma-Aldrich; Merck Millipore), into three different LMWF solutions as previously described (18). Fucoidan was dissolved in dimethyl sulfoxide $(10 \mathrm{mg} / \mathrm{ml})$, filtered using a $0.2 \mathrm{~mm}$ filter and stored at $-20^{\circ} \mathrm{C}$. The effect of these LMWF solutions on the growth and viability of osteoclasts was evaluated using MTT assay as previously described (19), in order to select the strongest inhibitory concentration as preparation for the subsequent experiments. The concentrations of the three LMWF solutions tested were 50, 10, 5, 1, 0.5 and $0.1 \mu \mathrm{g} / \mathrm{ml}$. It was determined that $5 \mu \mathrm{g} / \mathrm{ml}$ of the LMWF generated by pectinase digestion exhibited the strongest inhibitory effect on osteoclasts (Fig. 1A). Based on these results, subsequent experiments were performed using $5 \mu \mathrm{g} / \mathrm{ml}$ of the LMWF generated by pectinase digestion.

Cell culture, induction and analysis. The RAW264.7 murine macrophage-like cell line was purchased from the Chinese National Platform of Experimental Cell Resources for Sci-Tech, Institute of Basic Medicine, Chinese Academy of Medical Sciences (Peking Union Medical College, Beijing, China). RAW264.7 cells were grown in high glucose Dulbecco's modified Eagle's medium (DMEM; ME100202P1; Gibco; Thermo Fisher Scientific, Inc., Waltham, MA, USA) supplemented with $10 \%$ heat-inactivated fetal bovine serum (12676011; Gibco; Thermo Fisher Scientific, Inc.) and 1\% penicillin-streptomycin, at $37^{\circ} \mathrm{C}$ in a humidified atmosphere of $95 \%$ air and $5 \% \mathrm{CO}_{2}$. The cells were cultured on sterile coverslips or osteologic slides, which were placed into 6-well culture dishes. Cells were inoculated at a density of $2 \times 10^{4} / \mathrm{ml}$. The medium was changed to high-glucose DMEM (ME100202P1; Gibco; Thermo Fisher Scientific, Inc.) supplemented with $10 \%$ fetal bovine serum (Gibco; Thermo Fisher Scientific, Inc.), with 100 ng/ml RANKL (Murine sRANK-Ligand; 31501; PeproTech, Inc., Rocky Hill, NJ, USA) and $30 \mathrm{ng} / \mathrm{ml}$ M-CSF (Murine M-CSF, 31502; PeproTech, Inc.) to induce differentiation $4 \mathrm{~h}$ after cell attachment. Cells were incubated at $37^{\circ} \mathrm{C}$, in a $5 \% \mathrm{CO}_{2}$ incubator with high humidity. The medium was changed every 2 days. After 6 days, cells were stained for tartrate-resistant acid phosphatase (TRAP) using the TRAP staining kit (Sigma-Aldrich; Merck Millipore) and the experiment was conducted according to the manufacturer's protocol. A light microscope (Olympus Corporation, Tokyo, Japan) was used to observe the number of TRAP-positive multinucleated cells ( $\geq 3$ nuclei). To visualize bone resorption pits, osteologic slides were fixed in $2.5 \%$ glutaraldehyde, washed by sonication in phosphate-buffered saline (PBS), dehydrated in a graded, ascending series of ethanol, sputter-coated with gold and palladium. Images were captured using a scanning electron microscope.

Scoring of TRAP-positive cells and analysis of bone resorption surface. To assess the effects of LMWF on osteoclast differentiation, RAW264.7 cells $\left(2 \times 10^{4} / \mathrm{ml}\right)$ were cultured in two groups; A control group and an experimental, LMWF-treated group. Differentiation was induced as aforementioned, with the exception that the experimental group was cultured in the presence of $5 \mu \mathrm{g} / \mathrm{ml} \mathrm{LMWF}$ in addition to RANKL and M-CSF. Cells were stained for TRAP and the number of TRAP-positive multinucleated cells ( $\geq 3$ nuclei) was scored after 6 days. Scoring was performed at a magnification x100 and 10 fields were randomly selected and scored, with the average calculated. Only cells that appeared pink or rose-red and contained $\geq 3$ nuclei were counted, as previously described (19). The experiment was repeated three times. For the bone resorption pit assay, cells were cultured in as aforementioned, with the exception that they were cultured on bovine cortical bone slices made in-house. Cells were cultured on 10 bone slices in each group. Within each bone slice, 10 non-overlapping areas were randomly selected and analyzed using Image-Pro Plus version 6.0 image analysis software (Media Cybernetics, Inc., Rockville, MD, USA) to evaluate bone resorption areas.

Effects of LMWF on osteoclast proliferation. Two groups of cells, as aforementioned, were cultured for 6 days. Subsequently, the cells $\left(1 \times 10^{6} / \mathrm{ml}\right)$ were collected, washed with PBS, and fixed with $75 \%$ ethanol for $5 \mathrm{~min}$ at room temperature. Fixed cells were resuspended in $1 \mathrm{ml}$ propidium iodide/Triton X-100 staining solution (Sigma-Aldrich; Merck Millipore) containing $0.2 \mathrm{mg}$ RNase A and were incubated at $37^{\circ} \mathrm{C}$ for $15 \mathrm{~min}$. Stained cells were subjected to flow cytometry using a Guava Flow Cytometer (model, EPICS\&reg; Merck Millipore) and Expo 32 software (Beckman Coulter, Inc., Brea, CA, USA) to calculate proliferation index (PI). $\mathrm{PI}=\left(\mathrm{S}+\mathrm{G}_{2} / \mathrm{M}\right) /\left(\mathrm{G}_{0} / \mathrm{G}_{1}+\mathrm{S}+\mathrm{G}_{2} / \mathrm{M}\right)$.

Reverse transcription-quantitative polymerase chain reaction (RT-qPCR) for gene expression detection. After 6 days of induction, cells were harvested for RNA extraction. Total RNA was isolated using TRIzol reagent (Invitrogen; Thermo Fisher Scientific, Inc.) The quality of RNA was assessed by agarose gel electrophoretic analysis. RANK, TRAP, matrix metallopeptidase-9 (MMP-9), NFATc1 and osteoclast-associated immunoglobulin-like receptor (OSCAR) expression levels were detected by qPCR using the Absolute ${ }^{\mathrm{TM}}$ Fast qPCR Master Mixes kit (Guangzhou Funeng Gene Co., Ltd., 
Guangzhou, China). The thermocycler conditions used were as follows: $96^{\circ} \mathrm{C}$ pre-denaturation for $\sim 5 \mathrm{~min} ; 94^{\circ} \mathrm{C}$ denaturation for $\sim 1 \mathrm{~min} ; 58^{\circ} \mathrm{C}$ annealing for $\sim 45 \mathrm{sec} ; 72^{\circ} \mathrm{C}$ extension for $\sim 2 \mathrm{~min}$, for a total of 35 cycles. cDNA was reverse-transcribed using the All-in-One ${ }^{\mathrm{TM}}$ First-Strand cDNA Synthesis kit (GeneCopoeia, Inc., Rockville, MD, USA) from the isolated RNA and GAPDH expression was used as an internal control. Primers were designed by Fulengen Co., Ltd. (Guangzhou, China). The primer sequences were as follows: GAPDH, forward (F) 5'-CGGAGTCAACGGATTTGGTCGTAT-3', reverse (R) 5'-AGCCTTCTCCATGGTGGTGAAGAC-3', fragment amplified, 96 bp; NFATc1, F 5'-TCCAAAGTCATTTTC GTGGA-3', R 5'-CTTTGCTTCCATCTCCCAGA-3'; MMP-9, F 5'-ACGACATAGACGGCATCCA-3', R 5'-GCTGTGGTT CAGTTGTGGTG-3'; TRAP, F 5'-AAATCACTCTTTAAG ACCAG-3', R 5'-TTATTGAATAGCAGTGACAG-3'; RANK, F 5'-CCAGGGGACAACGGAATCA-3', R 5'-GGCCGG TCCGTGTACTCATC-3'; and OSCAR, F 5'-TGATTGGCA CAGCAGGAG-3' and R 5'-AAGGCACAGGAAGGAAAT AGAG-3'. RT and qPCR were performed per manufacturer's protocol. Amplification and melting curves were evaluated for each qPCR analysis. Gene expression levels were based on levels of the initial template, and were calculated using the $2^{-\Delta \Delta C q}$ method (20).

Animals. A total of 60 healthy 6-month-old female Sprague-Dawley (SD) rats weighing 220-240 g were provided by Tianjin Hospital Center for Experimental Animals (Tianjin, China; approval no. SCXK 2007004). All experimental procedures were approved by the Institutional Animal Care and Use Committee at the Tianjin Hospital Ethics Committee of China (Tianjin, China). The methods were carried out in accordance with the approved guidelines. Animals were housed at a room temperature of $21-24^{\circ} \mathrm{C}$, in a lighted room between 7:00 a. m. and 7:00 p.m.; animals were allowed to engage in physical activity and received ad libitum access to food and water.

Animal experiments. A total of 60 female SD rats were randomly assigned to the following treatment groups ( $n=20 /$ group): i) Ovariectomized group (OVX); ii) sham surgery group (SHAM); and iii) LMWF group (LMWF dose: $5 \mathrm{mg} / \mathrm{kg}$ ). SHAM group rats were subjected to a sham surgery (only an incision in the back was made) without ovary removal. The other two groups were subjected to bilateral ovary-removal surgery through an incision in the back following anesthesia with $10 \%$ chloral hydrate $(0.3 \mathrm{ml} / 100 \mathrm{~g})$. Saline (for the OVX and SHAM groups) or LMWF (for the LMWF group) was administered by intraperitoneal injection 5 days after surgery. Rats were euthanized with $10 \%$ chloral hydrate $(1 \mathrm{ml} / 100 \mathrm{~g})$ at 4 or 8 weeks following administration of treatment. A total of 10 rats from each group were euthanized at each time-point.

The bone mineral density (BMD) of the right femur of the rats ( $n=5$ per group at each time-point) was measured using LUNAR DPXIQ bone densitometer (GE Healthcare Bio-Sciences, Pittsburgh, PA, USA). Bones were then subjected to a micro-computed tomography (CT) scan (Bruker microCT, Antwerp, Belgium). Scanning was performed at a setting of $21-\mu \mathrm{m}$ resolution, $360^{\circ}$ rotation with an incremental rotation of $0.4^{\circ}$, voltage of $80 \mathrm{kV}$, current of $80 \mathrm{~A}$ and $2.960 \mathrm{msec}$ exposure time. Images were captured at an average gain of 4 , pixel $1 \times 1$.
For image normalization, the images were scanned in black and white and the grids were standardized. Trabecular bone volume fraction (BV/TV,\%), trabecular thickness (Tb.Th.) and trabecular number (Tb.N.) of the distal end of the femur were determined for evaluation.

For the 4-week and 8-week time-points, rats were injected with an intramuscular injection of tetracycline (25 $\mathrm{mg} / \mathrm{kg}$ ) at 10 days or 3 days prior to euthanasia, as previously described (21). Following euthanasia, the right femurs were collected and fixed in ethanol for 1 week. The distal $1 / 3$ of each femur ( $n=5$ per group at each time-point) was dehydrated and embedded in semi-butyl methacrylate polymerization/methyl ester. Using a heavy-duty microtome, $7 \mu \mathrm{m}$ undecalcified bone sections were cut. The sections were washed in distilled water and treated with $3 \%$ sodium thiosulphate for $5 \mathrm{~min}$, and then washed in water. von Kossa and Giemsa staining were performed for $5 \mathrm{~min}$, respectively, and sections were the mounted. Images were captured using a light microscope and analyzed using Image-Pro Plus version 6.0 software.

Rats were anesthetized with $10 \%$ chloral hydrate $(0.3 \mathrm{ml} / 100 \mathrm{~g})$ and serum samples $(1 \mathrm{ml})$ were collected by cardiac puncture 8 weeks after the administration of LMWF or saline control. Samples were centrifuged at $300 \mathrm{x} \mathrm{g}$ for $10 \mathrm{~min}$ at room temperature. Serum procollagen type I N propeptide (PINP) and C-terminal telopeptide-1 (CTX-1) levels were determined using corresponding ELISA kits (Ra PINP kit; cat. no. 20131010 and Ra CTX-1 kit; cat. no. 20130311; Cusabio Biotech Co., Ltd., Baltimore, MD, USA).

Assessment offemoralmechanical properties. The mechanical strength of the shaft and neck of the left femur ( $n=10 /$ group) was determined as previously described by Ma and Fu (22). All testing was performed on a Bose ElectroForce3200 electromagnetic test instrument (Bose Corporation, Framingham, MA, USA). The midshaft femoral strength was tested using a three-point bending test and the femoral neck strength was tested by a compression test $(n=10)$.

Statistical analysis. Data are presented as the mean \pm standard deviation. Statistical analysis was conducted using SPSS 16.0 software (SPSS, Inc., Chicago, IL, USA). All experimental data were analyzed using an one way analysis of variance and multiple comparisons were performed using Duncan's test. $\mathrm{P}<0.05$ was considered to indicate a statistically significant difference.

\section{Results}

Number of TRAP-positive cells and analysis of the bone resorption surface. As presented in Fig. 1B, 6 days after RANKL induction of RAW264.7 cells, the number of cells that stained positive for TRAP in the LMWF group was significantly lower compared with the control group $(\mathrm{P}<0.05)$, indicating that more osteoclasts were generated when LMWF was absent. In addition, TRAP-positive cells from the LMWF group had fewer nuclei in general $(\mathrm{P}<0.05$; data not shown). The bone resorption assay revealed that there were significantly more bone resorption pits in the control group compared with the LMWF group ( $\mathrm{P}<0.05$; Fig. $1 \mathrm{C})$. 


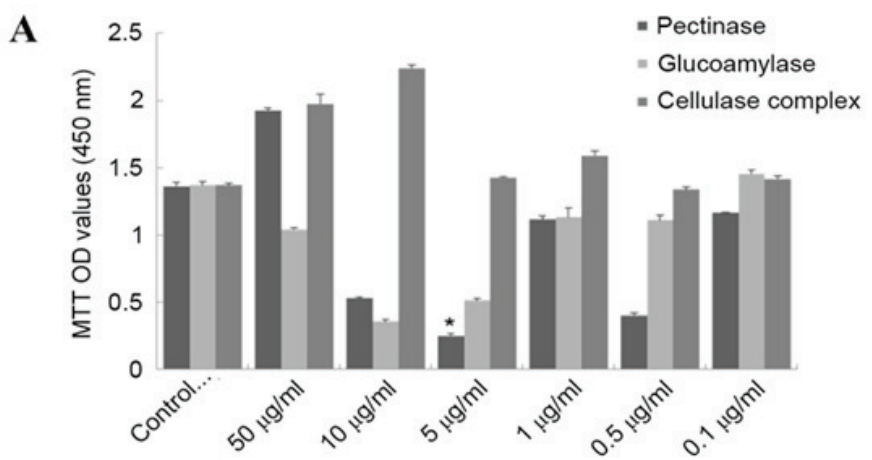

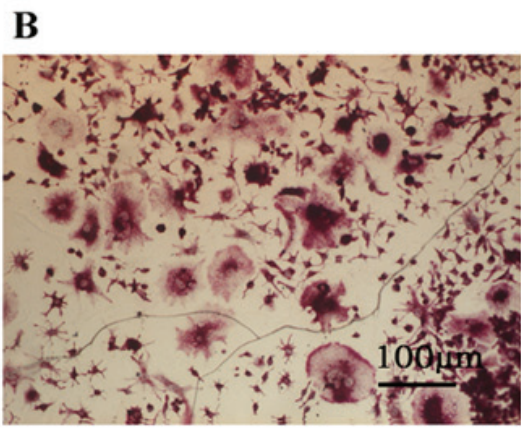

C

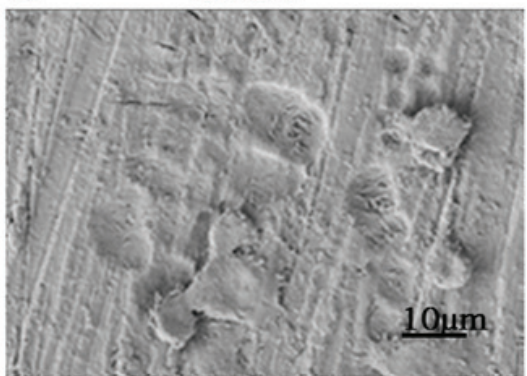

Control

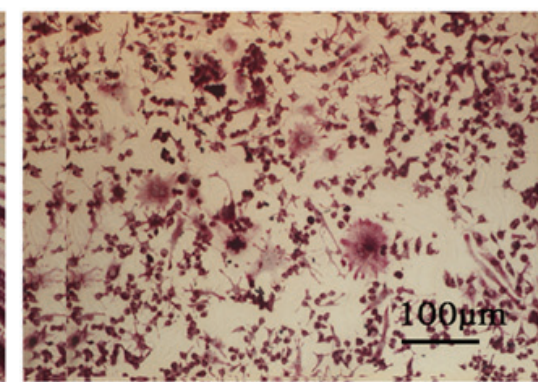

LMWF

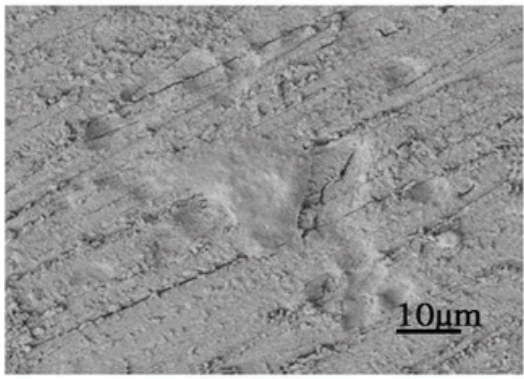

LMWF
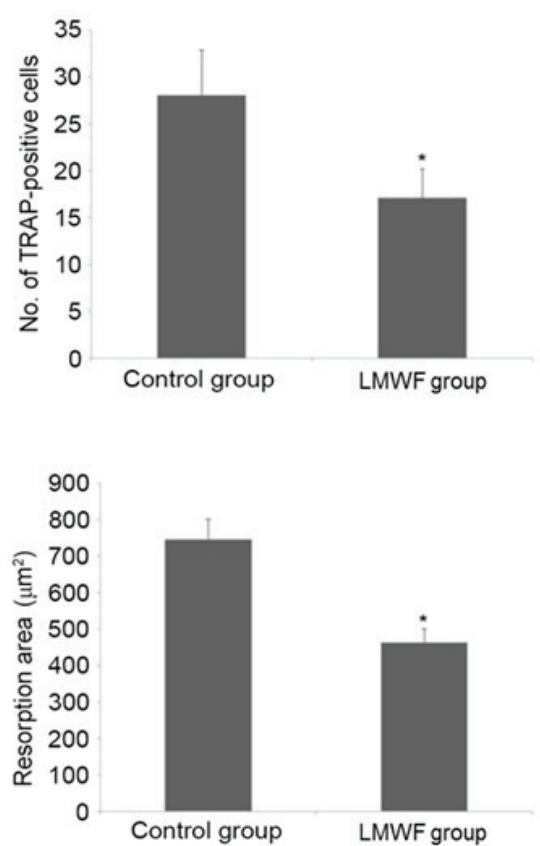

Figure 1. (A) MTT assay quantification. (B) Quantification of the TRAP-positive cells. (C) Analysis of bone resorption surface. "P<0.05 vs. control. OD, optical density; TRAP, tartrate-resistant acid phosphatase.

Effects of LMWF on osteoclast proliferation. FACS analysis revealed that the PI of cells from the LMWF group was significantly lower compared with cells from the control group (26.2 \pm 3.62 vs. $37.56 \pm 3.08$, respectively; $\mathrm{P}<0.05$; data not shown), thus suggesting that LMWF inhibited osteoclast proliferation.

Effects of LMWF on expression of genes involved in osteoclast differentiation. RT-qPCR was performed to assess expression levels of the genes encoding TRAP, RANK, NFATc1, OSCAR and MMP-9. As presented in Fig. 2, LMWF significantly inhibited the expression levels of osteoclast marker genes TRAP $(\mathrm{P}<0.05)$, NFATc1 $(\mathrm{P}<0.01)$ and OSCAR $(\mathrm{P}<0.05)$ when compared with the control group. However, LMWF had no effect on RANK gene expression $(\mathrm{P}>0.05)$. LMWF also exhibited an inhibitory effect on MMP-9 expression $(\mathrm{P}<0.05)$.

$B M D$ and micro-CT analysis in OVX rats. As presented in Fig. 3A, 4 weeks after the ovariectomy the distal femur of the OVX group exhibited trabecular bone loss, which progressively worsened 8 weeks after surgery when compared with the SHAM group. As presented in Fig. 3B, the BMD of rats from the OVX group was significantly lower for both time-points investigated compared with that of rats from the SHAM group $(\mathrm{P}<0.05)$, thus suggesting that ovariectomy may lead to bone loss. The ovariectomized rats receiving LMWF had a significantly higher BMD compared with the OVX group $(\mathrm{P}<0.05$; Fig. 3B), indicating that LMWF may minimize bone loss. In addition, the BMD increase at the 8-week time-point was higher than that at the 4-week time-point; however, still lower when compared with the SHAM group.

Quantification of micro-CT scan results are presented in Fig. 3C-E. Compared with the OVX group, the LMWF group demonstrated a significantly higher BV/TV, Tb.Th. and Tb.N at 8 weeks ( $\mathrm{P}<0.05$ for all measurements), whereas these measurement were lower compared with in the SHAM group. In addition, the rats of the LMWF group demonstrated greater Tb.N in the distal femur compared with the OVX group.

Histomorphometric analysis. Fig. 4 presents the quantitative histomorphometric analyses of the undecalcified histological sections stained with (A) von Kossa and (B) Giemsa. The OVX group exhibited higher tetracycline labeling (Fig. 4C), thicker osteoid seams, and a higher rate of mineralization on osteoblast surfaces and osteoclast surfaces when compared 

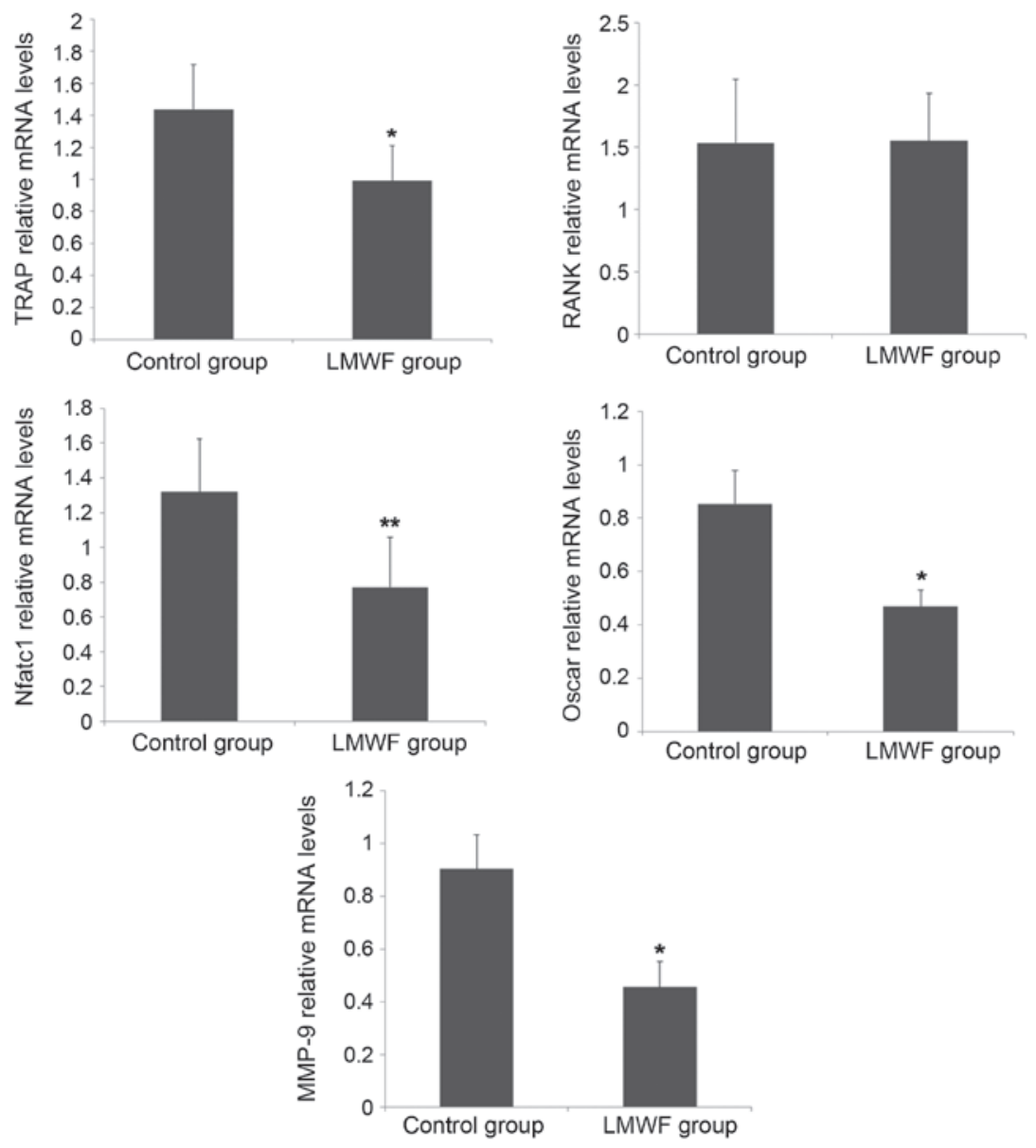

Figure 2. Reverse transcription-quantitative polymerase chain reaction analysis of osteoclast-specific transcripts to assess the effect of LMWF on osteoclast differentiation. ${ }^{*} \mathrm{P}<0.05,{ }^{* * *} \mathrm{P}<0.01$ vs. control. LMWF, low-molecular weight fucoidan; TRAP, tartrate-resistant acid phosphatase; RANK, receptor activator of nuclear factor $\mathrm{\kappa B}$; Nfatc1, nuclear activator of activated T-cells 1; OSCAR, osteoclast-associated immunoglobulin-like receptor; MMP-9, matrix metallopeptidase-9.

with the SHAM group (Fig. 4D; P<0.05), which was consistent with the high bone turnover usually observed in osteoporosis. LMWF administration reduced bone turnover rate in OVX rats; however, the surface area for bone formation and the mineralization time was increased compared with the OVX group (Fig. 4D; $\mathrm{P}<0.05$ ).

Mechanical testing. As presented in Fig. 5A, the maximal strength of the femur as assessed by the three-point bending test and compression test was significantly lower in the OVX group when compared with the SHAM group rats $(\mathrm{P}<0.05)$. The maximum load of femoral neck in the OVX group was significantly reduced 8 weeks after surgery when compared with 4 weeks after surgery $(\mathrm{P}<0.05)$. Mechanical strength of femoral neck and mid-shaft femoral in OVX rats was lower compared with the SHAM group ( $\mathrm{P}<0.05$; Fig. 5A). The LMWF group demonstrated higher femoral mechanical strength of femoral neck and mid-shaft femoral in rats 4 and 8 weeks after ovariectomy when compared with the OVX group $(\mathrm{P}<0.05$; Fig. 5A).

Serum analysis. The OVX group had significantly increased levels of CTX-1 and PINP in serum compared with the SHAM group rats at 4 and 8 weeks after the operation. As presented in Fig. 5B LMWF treatment significantly decreased the levels of serum PINP $(\mathrm{P}<0.01$ for both time-points) and CTX-1 $(\mathrm{P}<0.01$ at 4 weeks and $\mathrm{P}<0.05$ at 8 weeks) compared with the OVX group rats.

\section{Discussion}

The present study determined that LMWF inhibits the induction of RAW264.7 cells into mature osteoclasts by RANKL and M-CSF in vitro. The in vivo experiments using an osteoporosis animal model confirmed that LMWF inhibited bone loss and prevented microarchitectural deterioration, and increased femoral mechanical strength following ovariectomy. To the best of our knowledge, the present study is the first to determine the potential use of LMWF for the treatment of osteoporosis.

M-CSF and RANKL are considered the key signaling factors in the process of osteoclast differentiation (9). RANKL is essential for osteoclastogenesis and the subsequent bone resorption (8), whereas M-CSF may stimulate the movement and expansion of osteoclasts, inhibit their apoptosis and enhance RANKL-induced osteoclast formation $(7,23)$. TRAP staining is a commonly used method to identify osteoclasts (24) and the present study revealed that following RANKL and M-CSF administration, TRAP-positive osteoclast-like cells were observed 2 days after the induction, and a greater number was observed 6 days after the induction. Examination of TRAP-stained slides and thin osteologic 
$\mathbf{A}$
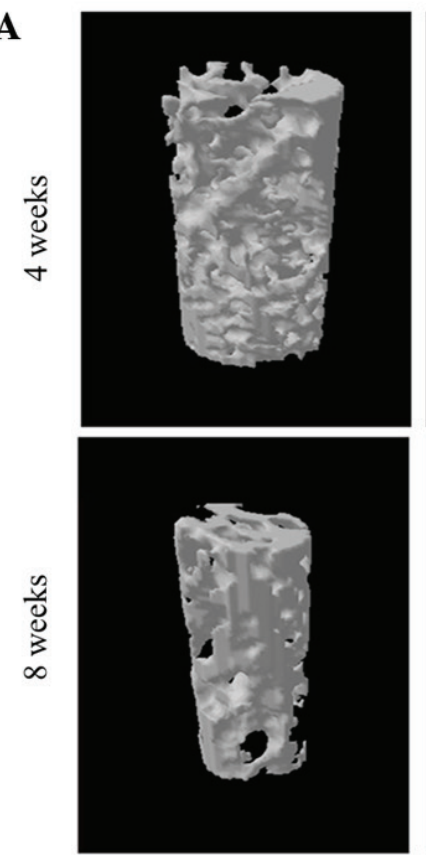

Sham
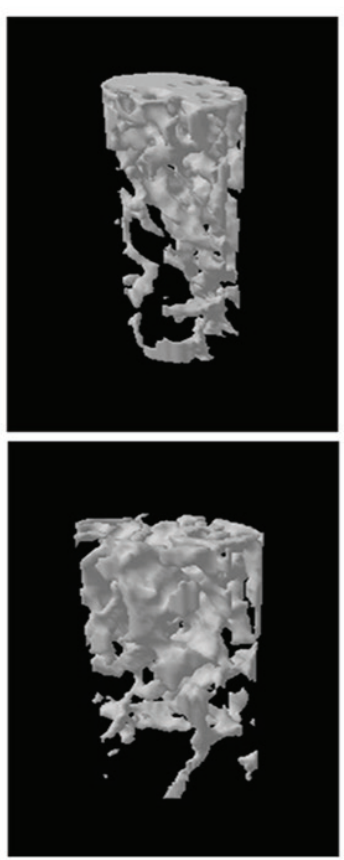

ovX
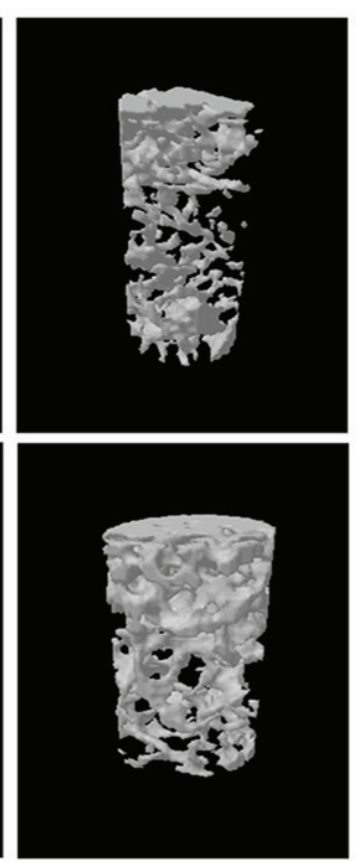

LMWF

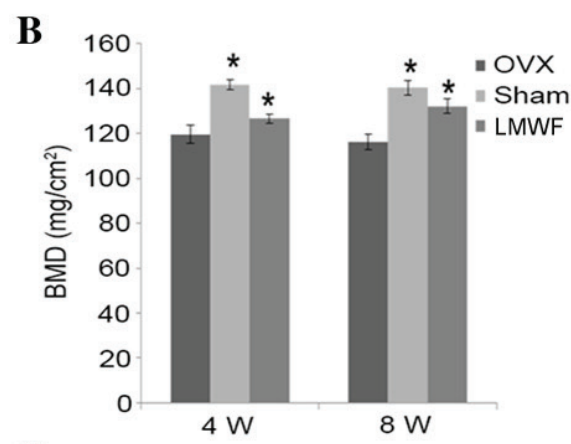

$\mathbf{C}$
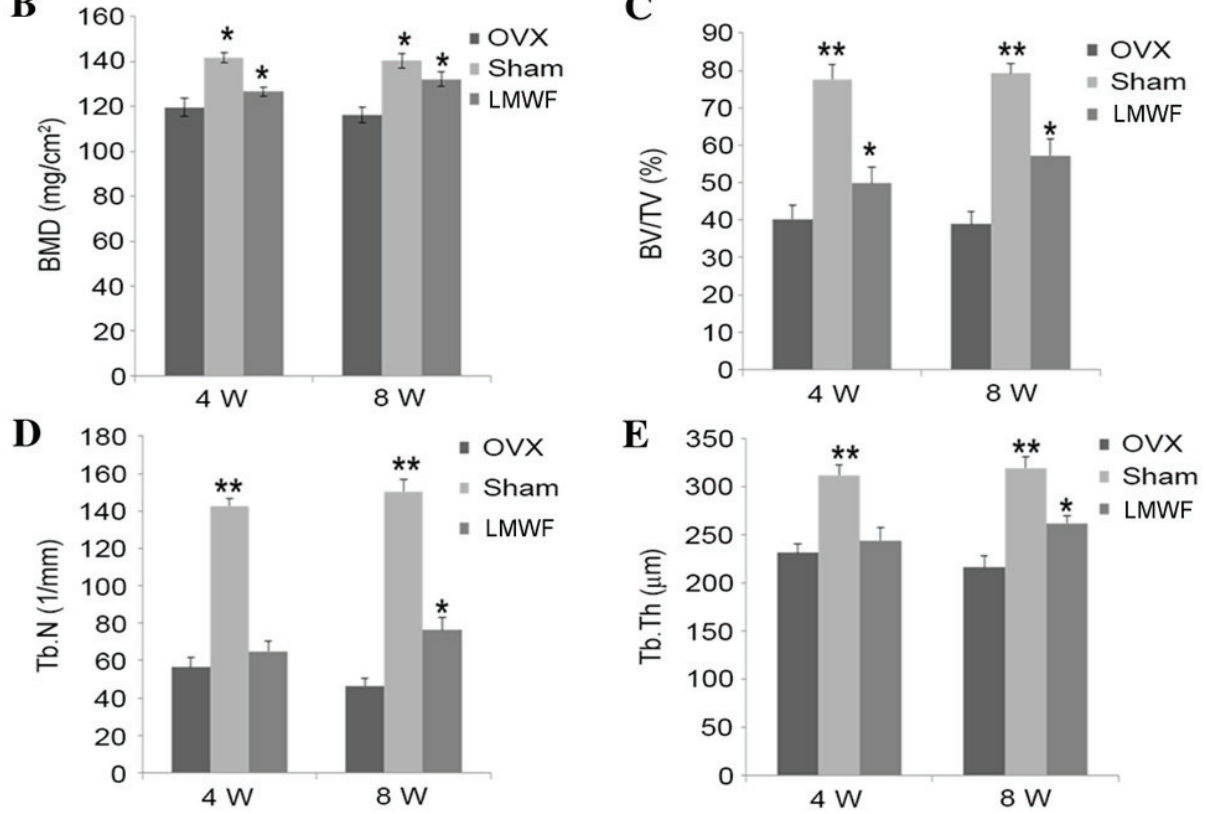

Figure 3. (A) Representative samples of 3D architecture of trabecular bone within the distal femoral metaphyseal region from the different treatment groups. (B) BMD quantification using dual energy X-ray absorptiometry. Effects of low-weight molecular fucoidan on the (C) BV/TV, (D) Tb.n and (E) Tb.Th of the distal femoral metaphysic in OVX rats by micro-computed tomography analysis. Data are presented as the mean \pm standard deviation, $\mathrm{n}=5$. ${ }^{*} \mathrm{P}<0.05,{ }^{* * *} \mathrm{P}<0.01$ vs. OVX group. OVX, ovariectomized; BMD, bone mineral density; BV/TV, bone volume/total volume; Tb.n, trabecular number; Tb.Th, trabecular thickness; $4 \mathrm{~W}, 4$ weeks; $8 \mathrm{~W}, 8$ weeks.

slides for bone resorption assay revealed that the presence of LMWF in addition to RANKL and M-CSF resulted in fewer mature osteoclasts and reduced bone resorption pits compared with the untreated control group.

MMP-9 is secreted by osteoclasts, and in addition to degradation of the matrix, aids in osteoclast movement $(25,26)$. The present study determined that LMWF reduced the expression levels of TRAP and MMP-9. NFATc1 is an important transcription factor for osteoclastogenesis, and is involved in the regulation of the expression of numerous osteoclast-specific genes; therefore, it has an impact on osteoclast cell formation and function $(27,28)$. Cytoplasmic NF- $\kappa \mathrm{B}$ is activated during osteoclast differentiation following RANKL stimulation and may result in activation of intracellular signaling cascades. The activated NF- $\kappa \mathrm{B}$ is subsequently translocated to the nucleus with NFATc2, to activate expression and auto-amplification of NFATc1 $(29,30)$. The present study revealed that LMWF may significantly inhibit NFATc1 expression in osteoclasts. RANK is a type I membrane receptor protein on osteoclasts and their precursor cells; the binding of RANK and RANKL 
$\mathbf{A}$

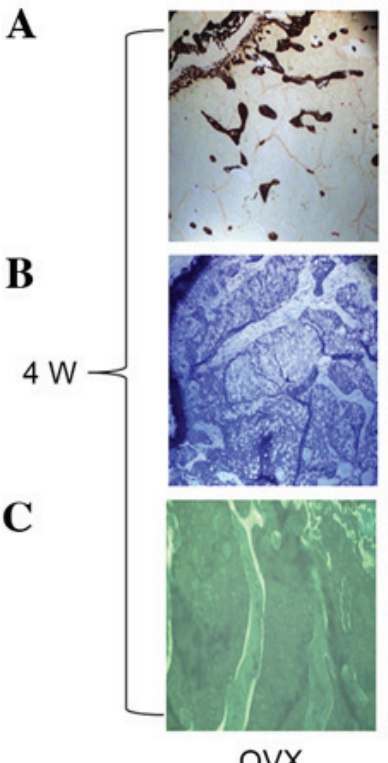

ovx
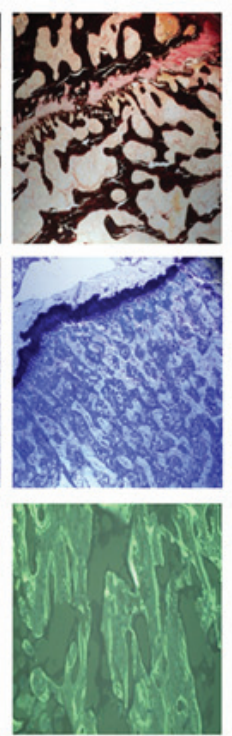

Sham
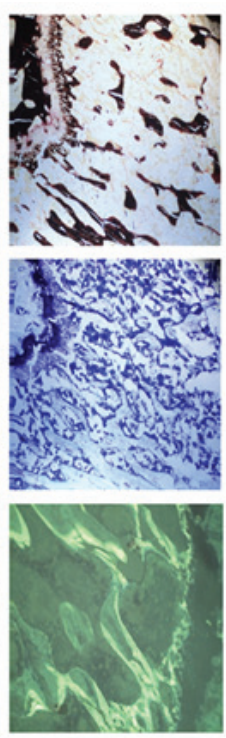

LMWF

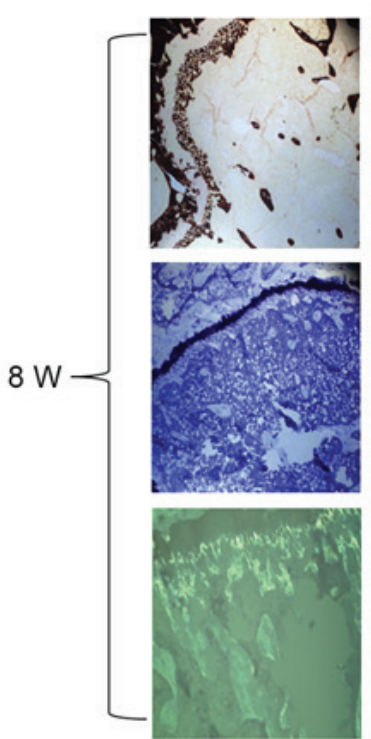

ovX
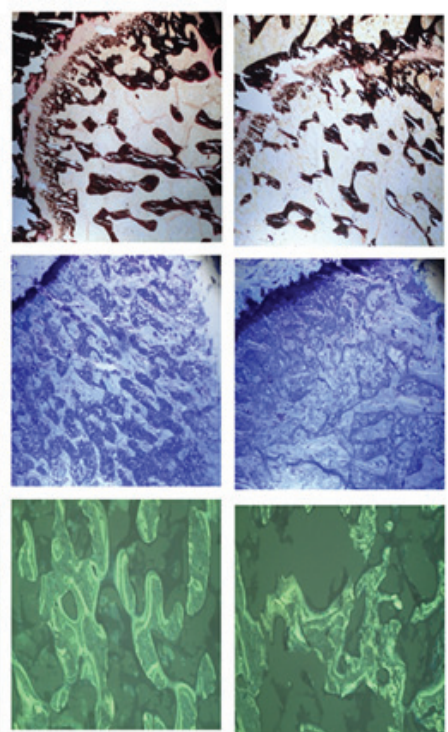

Sham

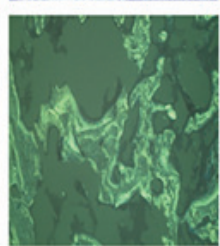

LMWF
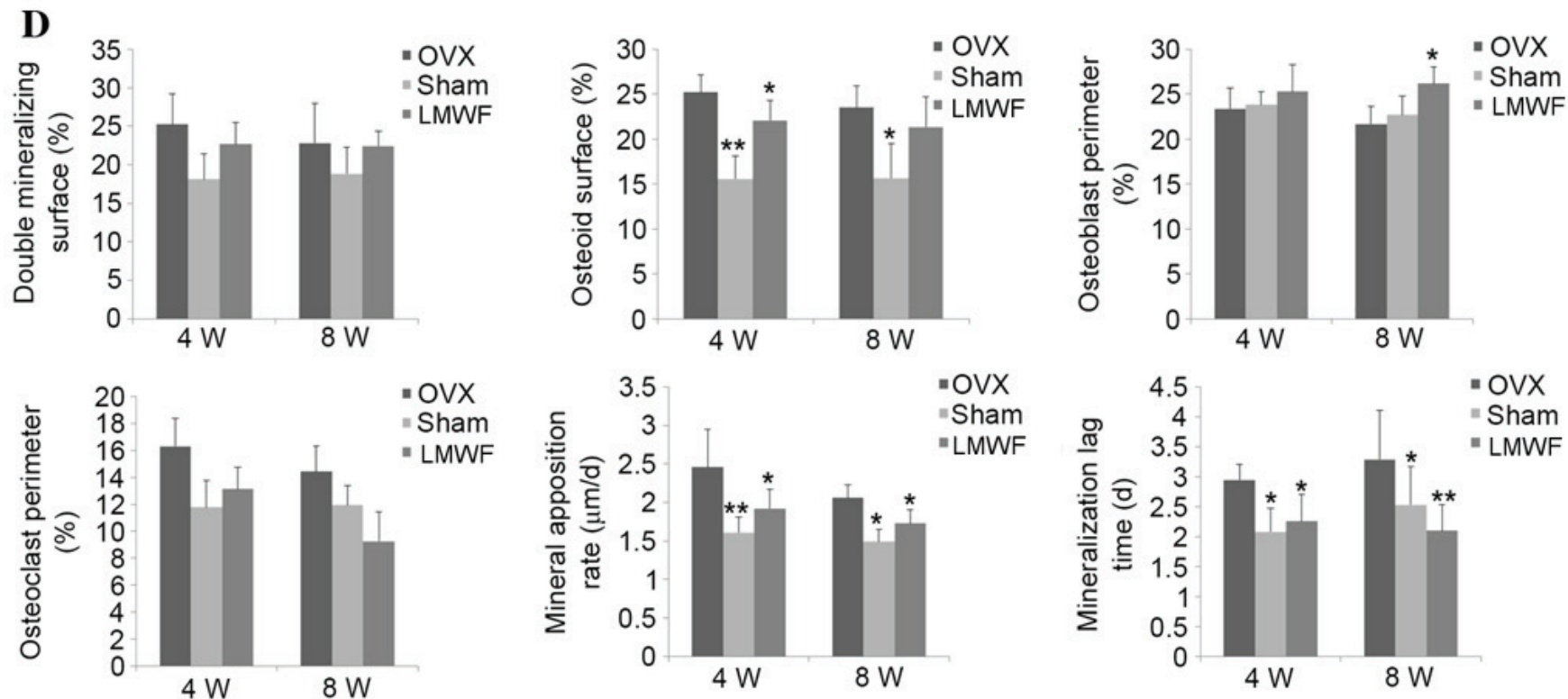

Figure 4. Undecalcified histological sections, stained with (A) von Kossa and (B) Giemsa, of the sagittal plane through the distal of femur. Magnification, x100. (C) Undecalcified sections observed under a fluorescence microscope showing tetracycline fluorescence. Magnification, $\mathrm{x} 200$. (D) Quantification using Image-Pro Plus version 6.0. Data are presented as the mean \pm standard deviation. ${ }^{*} \mathrm{P}<0.05,{ }^{* *} \mathrm{P}<0.01$ vs. OVX group. OVX, ovariectomized; $4 \mathrm{~W}, 4$ weeks; $8 \mathrm{~W}$, 8 weeks.

subsequently activates the signal transduction pathway in precursor cells, resulting in osteoclast maturation. In mature osteoclasts, this activated pathway serves to maintain bone resorption activity and survival of osteoclasts (31). In the present study, LMWF had no effect on RANK mRNA expression. Therefore, it is also possible that it does not affect osteoclast cell membrane RANK receptors. OSCAR is an osteoclast-specific receptor that was recently identified (32) and is a type of leukocyte receptor that may be an important factor in inducing osteoclastogenesis $(32,33)$. The present study determined that LMWF inhibited OSCAR mRNA expression; therefore, it may also inhibit RANKL- and M-CSF-induced differentiation of RAW264.7 cells to osteoclasts, thus reducing the number of osteoclasts and the rate of bone resorption.

RANKL-induced activation of the $N F-\kappa B$ pathway is a key factor in osteoclast differentiation (34). NFATc1 is likely to be the key factor regulating RANKL-induced osteoclast differentiation, cell fusion and activation (28). $\mathrm{NF}-\kappa \mathrm{B}$ induction of NFATc1 is important and may occur via $\mathrm{NF}-\kappa \mathrm{B}$ binding to the promoter region of NFATc1, leading to increased NFATc1 expression and subsequently, RANKL-induced osteoclast differentiation (35). The present study revealed that LMWF inhibited RANKL-induced osteoclast formation.

In the present study, the in vivo consequences of the in vitro observations were investigated in an ovariectomized rat osteoporosis model. The effects of LMWF on BMD, bone microarchitecture, remodeling and mechanical competence were investigated. The results demonstrated that 4 weeks after treatment with LMWF, BMD was higher in the LMWF group when compared with the OVX group, and 8 weeks after surgery BMD increased further. 

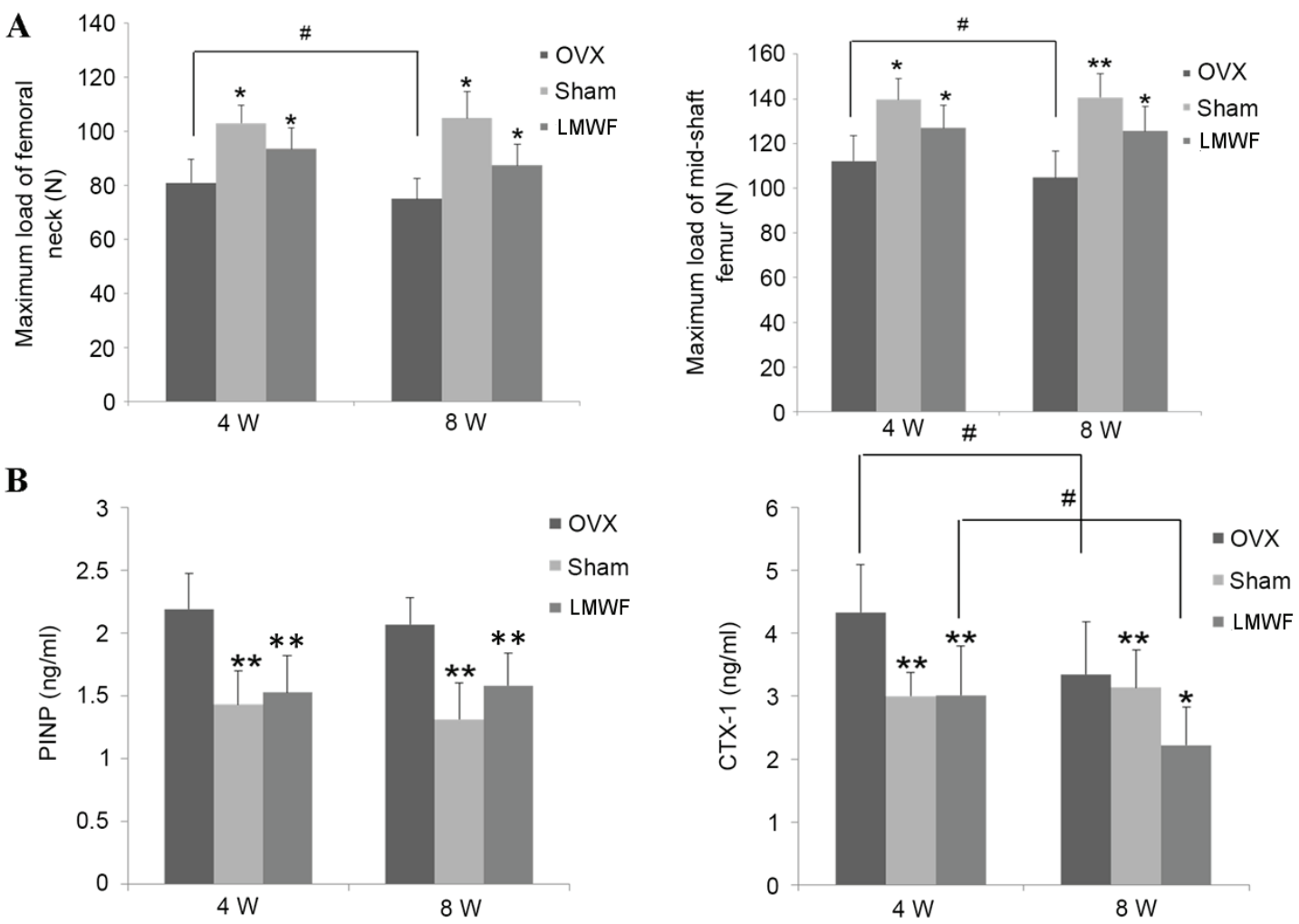

Figure 5. (A) Effect of low-weight molecular fucoidan on the mechanical strength of the mid-shaft femur and femoral neck of OVX rats (n=5), with data expressed in Newtons. (B) Serum analysis of PINP and CTX-1, $\mathrm{n}=8$ samples/group. Data are presented as the mean \pm standard deviation. ${ }^{*} \mathrm{P}<0.05$, ${ }^{* * *} \mathrm{P}<0.01$ vs. OVX group; "P<0.05. OVX, ovariectomized; PINP, procollagen type I N propeptide; CTX-1, C-terminal telopeptide-1; 4 W, 4 weeks; 8 W, 8 weeks.

BMD has been identified as an important factor for the evaluation of osteoporosis-related fractures; a previous study revealed that in accordance with BMD criteria, only $10-53 \%$ of patients are diagnosed with osteoporosis following an osteoporotic fracture (36). Micro-CT voxel-based test unit may be used for the early detection of lesions and bone structure deterioration $(37,38)$. The micro-CT scans performed in the present study revealed that the trabecular microarchitecture in the distal femur of ovariectomized rats was deteriorated 4 weeks after surgery, whereas the SHAM group exhibited no significant changes. However, the parameters of trabecular microarchitecture were all improved in the LMWF group compared with the OVX group. When the treatment time was extended to 8 weeks following surgery, BV/TV and Tb.N. exhibited further improvement. This may suggest that LMWF prevented bone loss due to ovariectomy and may also promote bone regeneration, and that bone microarchitectural recovery may be directly associated with treatment duration. The 3D reconstituted distal femur revealed that in the OVX group, the quantity of trabecular bone and connectivity were decreased and trabecular bone separation increased when compared with the SHAM group. In the LMWF group, the quantity of trabecular bone increased, spacing narrowed and connectivity recovered. Histophotometry was used in order to evaluate bone structure further.

LMWF it inhibited high bone turnover rate, which is usually the result of an ovariectomy, increased the osteoid and bone formation surface areas (osteoblast perimeter), decreased the surface for bone resorption (osteoclast perimeter), inhibited mineralization, and increased mineralization time after ovariectomy. Collectively, these results suggested that LMWF inhibited the high bone turnover rate and bone loss associated with ovariectomy.

Consistent with the histomorphometry results, it was demonstrated that the OVX group had increased bone turnover, since the levels of PINP and CTX-1 were increased, this was also in line with a previous study (39). LMWF attenuated the increase in PINP and CTX-1 serum levels, thus suggesting that LMWF exerts its effects on bone metabolism by inhibiting bone turnover.

The three-point bending test and the femoral neck compression test were used to evaluate the effects of LMWF on the mechanical strength of the femoral shaft and femoral neck. The present study determined that 4 weeks after ovariectomy, the mechanical strength of femurs in the OVX group was significantly reduced. The mechanical strength in the OVX group at the 8-week time-point was lower than that at 4 weeks, which may be a result of weight gain 8 weeks after surgery, this phenomenon is itself likely a self-protective mechanism in which bone mass is augmented (40). The mechanical strength of the bones from the LMWF group was significantly increased 4 weeks after the surgery when compared with the OVX group. The 8-week time-point had a further increase in mechanical strength. Therefore, it is evident that LMWF may increase the mechanical strength of the femoral shaft and neck of ovariectomized rats.

In conclusion, LMWF may inhibit osteoclast precursor differentiation, osteoclast maturation and bone resorption, ameliorate loss of BMD and trabecular deterioration, and prevent loss of mechanical competence. These findings 
suggested that LMWF may be a novel therapeutic agent for the treatment of postmenopausal osteoporosis. Additional research is required to determine the optimal dosage, length of treatment and expected duration of effect for each dose administered.

\section{References}

1. Melton LJ III, Chrischilles EA, Cooper C, Lane AW and Riggs BL: How many women have osteoporosis? JBMR Anniversary Classic. JBMR, Volume 7, Number 9, 1992. J Bone Miner Res 20: 886-892, 2005.

2. Sowers MR, Zheng H, Jannausch ML, McConnell D, Nan B, Harlow S and Randolph JF Jr: Amount of bone loss in relation to time around the final menstrual period and follicle-stimulating hormone staging of the transmenopause. J Clin Endocrinol Metab 95: 2155-2162, 2010

3. Ritchie RO, Buehler MJ and Hansma P: Plasticity and toughness in bone. Phys Today 62: 41-47, 2009.

4. Zimmermann EA, Schaible E, Bale H, Barth HD, Tang SY, Reichert P, Busse B, Alliston T, Ager JW III and Ritchie RO: Age-related changes in the plasticity and toughness of human cortical bone at multiple length scales. Proc Natl Acad Sci USA 108: 14416-14421, 2011

5. Fitzpatrick P, Kirke PN, Daly L, Van Rooij I, Dinn E, Burke H, Heneghan J, Bourke G and Masterson J: Predictors of first hip fracture and mortality post fracture in older women. Ir J Med Sci 170: 49-53, 2001.

6. Matsuo K: Cross-talk among bone cells. Curr Opin Nephrol Hypertens 18: 292-297, 2009.

7. Teitelbaum SL: Bone resorption by osteoclasts. Science 289 : 1504-1508, 2000

8. Wada T, Nakashima T, Hiroshi $\mathrm{N}$ and Penninger JM: RANKL-RANK signaling in osteoclastogenesis and bone disease. Trends Mol Med 12: 17-25, 2006.

9. Asagiri $\mathrm{M}$ and Takayanagi $\mathrm{H}$ : The molecular understanding of osteoclast differentiation. Bone 40: 251-264, 2007.

10. Amano H, Yamada S and Felix R: Colony-stimulating factor-1 stimulates the fusion process in osteoclasts. J Bone Miner Res 13: 846-853, 1998

11. Rachner TD, Khosla S and Hofbauer LC: Osteoporosis: Now and the future. Lancet 377: 1276-1287, 2011.

12. Boonen S, Reginster JY, Kaufman JM, Lippuner K, Zanchetta J, Langdahl B, Rizzoli R, Lipschitz S, Dimai HP, Witvrouw R, et al: Fracture risk and zoledronic acid therapy in men with osteoporosis. N Engl J Med 367: 1714-1723, 2012.

13. Jeong HS, Venkatesan J and Kim SK: Hydroxyapatite-fucoidan nanocomposites for bone tissue engineering. Int J Biol Macromol 57: 138-141, 2013.

14. Thinh PD, Menshova RV, Ermakova SP, Anastyuk SD, Ly BM and Zvyagintseva TN: Structural characteristics and anticancer activity of fucoidan from the brown alga sargassum mcclurei. Mar Drugs 11: 1456-1476, 2013.

15. Changotade S, Korb G, Bassil J, Barroukh B, Willig C, Colliec-Jouault S, Durand P, Godeau G and Senni K: Potentia effects of a low-molecular-weight fucoidan extracted from brown algae on bone biomaterial osteoconductive properties. J Biomed Mater Res A 87: 666-675, 2008

16. Park HS, Hwang HJ, Kim GY, Cha HJ, Kim WJ, Kim ND, Yoo YH and Choi YH: Induction of apoptosis by fucoidan in human leukemia U937 cells through activation of p38 MAPK and modulation of Bcl-2 family. Mar Drugs 11: 2347-2364, 2013

17. Park SB, Chun KR, Kim JK, Suk K, Jung YM and Lee WH: The differential effect of high and low molecular weight fucoidans on the severity of collagen-induced arthritis in mice. Phytother Res 24: 1384-1391, 2010.

18. Yan-li C, Pei-pei W, Jun-ming Z, Song-song Z and Jian-wei: Extraction of fucoidan using multienzyme enzymolysis and their structural analysis, antioxidation research. Journal of Zhejiang University (Science Edition) 38: 536-540, 2011 (In Chinese).

19. Mar Arriero M, Ramis JM, Perelló J and Monjo M: Inositol Hexakisphosphate inhibits osteoclastogenesis on RAW 264.7 cells and human primary osteoclasts. PloS One 7: e43187, 2012.

20. Livak KJ and Schmittgen TD: Analysis of relative gene expression data using real-time quantitative PCR and the 2(-Delta Delta C(T)) method. Methods 25: 402-408, 2001.
21. Dalle Carbonare L, Valenti MT, Bertoldo F, Zanatta M, Zenari S, Realdi G, Lo Cascio V and Giannini S: Bone microarchitecture evaluated by histomorphometry. Micron 36: 609-616, 2005.

22. $\mathrm{Ma} \mathrm{Z}$ and $\mathrm{Fu} \mathrm{Q}$ : Comparison of the therapeutic effects of yeast-incorporated gallium with those of inorganic gallium on ovariectomized osteopenic rats. Biol Trace Elem Res 134: 280-287, 2010.

23. Lum L, Wong BR, Josien R, Becherer JD, Erdjument-Bromage H, Schlöndorff J, Tempst P, Choi Y and Blobel CP: Evidence for a role of a tumor necrosis factor-alpha (TNF-alpha)-converting enzyme-like protease in shedding of TRANCE, a TNF family member involved in osteoclastogenesis and dendritic cell survival. J Biol Chem 274: 13613-13618, 1999.

24. Cole AA and Walters LM: Tartrate-resistant acid phosphatase in bone and cartilage following decalcification and cold-embedding in plastic. J Histochem Cytochem 35: 203-206, 1987.

25. Chakrabarti S and Patel KD: Matrix metalloproteinase-2 (MMP-2) and MMP-9 in pulmonary pathology. Exp Lung Res 31: 599-621, 2005.

26. Delaissé JM, Engsig MT, Everts V, del Carmen Ovejero M, Ferreras M, Lund L, Vu TH, Werb Z, Winding B, Lochter A, et al: Proteinases in bone resorption: Obvious and less obvious roles. Clinica Chimica Acta 291: 223-234, 2000.

27. Ishida N, Hayashi K, Hoshijima M, Ogawa T, Koga S, Miyatake Y, Kumegawa M, Kimura T and Takeya T: Large scale gene expression analysis of osteoclastogenesis in vitro and elucidation of NFAT2 as a key regulator. J Biol Chem 277: 41147-41156, 2002.

28. Asagiri M, Sato K, Usami T, Ochi S, Nishina H, Yoshida H, Morita I, Wagner EF, Mak TW, Serfling E and Takayanagi H: Autoamplification of NFATc1 expression determines its essential role in bone homeostasis. J Exp Med 202: 1261-1269, 2005.

29. Matsuo K, Galson DL, Zhao C, Peng L, Laplace C, Wang KZ, Bachler MA, Amano $\mathrm{H}$, Aburatani $\mathrm{H}$, Ishikawa $\mathrm{H}$ and Wagner EF: Nuclear factor of activated T-cells (NFAT) rescues osteoclastogenesis in precursors lacking c-Fos. J Biol Chem 279: 26475-26480, 2004.

30. Zhao Q, Wang X, Liu Y, He A and Jia R: NFATc1: Functions in osteoclasts. Int J Biochem Cell Biol 42: 576-579, 2010.

31. Kong YY, Yoshida H, Sarosi I, Tan HL, Timms E, Capparelli C, Morony S, Oliveira-dos-Santos AJ, Van G, Itie A, et al: OPGL is a key regulator of osteoclastogenesis, lymphocyte development and lymph-node organogenesis. Nature 397: 315-323, 1999.

32. Nemeth K, Schoppet M, Al-Fakhri N, Helas S, Jessberger R, Hofbauer LC and Goettsch C: The role of osteoclast-associated receptor in osteoimmunology. J Immunol 186: 13-18, 2011.

33. Barrow AD, Raynal N, Andersen TL, Slatter DA, Bihan D, Pugh N, Cella M, Kim T, Rho J, Negishi-Koga T, et al: OSCAR is a collagen receptor that costimulates osteoclastogenesis in DAP12-deficient humans and mice. J Clin Invest 121: 3505-3516, 2011.

34. Yu M, Qi X, Moreno JL, Farber DL and Keegan AD: NF-кB signaling participates in both RANKL-and IL-4-induced macrophage fusion: Receptor cross-talk leads to alterations in NF- $\kappa B$ pathways. J Immunol 187: 1797-1806, 2011.

35. Liou SF, Hsu JH, Lin IL, Ho ML, Hsu PC, Chen LW, Chen IJ and Yeh JL: KMUP-1 suppresses RANKL-induced osteoclastogenesis and prevents ovariectomy-induced bone loss: Roles of MAPKs, Akt, NF- $\kappa$ B and calcium/calcineurin/NFATc1 pathways. PloS One 8: e69468, 2013.

36. McNamara LM: Perspective on post-menopausal osteoporosis: Establishing an interdisciplinary understanding of the sequence of events from the molecular level to whole bone fractures. J R Soc Interface 7: 353-372, 2010.

37. Brouwers JEM, Lambers FM, Gasser JA, van Rietbergen B and Huiskes R: Bone degeneration and recovery after early and late bisphosphonate treatment of ovariectomized wistar rats assessed by in vivo micro-computed tomography. Calcif Tissue Int 82 : 202-211, 2008.

38. Boyd SK, Davison P, Müller R and Gasser JA: Monitoring individual morphological changes over time in ovariectomized rats by in vivo micro-computed tomography. Bone 39: 854-862, 2006.

39. Yoon KH, Cho DC, Yu SH, Kim KT, Jeon Y and Sung JK: The change of bone metabolism in ovariectomized rats: Analyses of microCT scan and biochemical markers of bone turnover. J Korean Neurosurg Soc 51: 323-327, 2012.

40. Wronski TJ, Schenk PA, Cintrón M and Walsh CC: Effect of body weight on osteopenia in ovariectomized rats. Calcif Tissue Int 40: 155-159, 1987. 